\title{
Adaptación a la realidad docente como oportunidad para la adquisición de competencias en el desarrollo de las sesiones de prácticas de la asignatura de Empresa en Ingeniería del Diseño
}

\section{Inmaculada Marqués ${ }^{a}$, Loreto Fenollosab y Inmaculada Guaita Pradas ${ }^{c}$}

aUniversitat Politècnica de València, Departamento de Economía y Ciencias Sociales (imarques@esp.upv.es) bUniversitat Politècnica de València, Departamento de Economís y Ciencias Sociales (maferi@esp.upv.es) y 'Univesitat Politècnica de València, Departamento de Economía y Ciencias Sociales (iguaita@esp.upv.es)

\begin{abstract}
The European Higher Education Area has introduced to a new organization of university education at different levels. With a changing in education orientation, from learning content, to an orientation based on learning and development competencies. The Design career is to train scientific and technical professionals to be able to direct and manage the entire life of a new product, from idea generation (marketing, basicdesign, ...) through production, manufacturing and product promoting, to the environmental impact assessment at the end of its useful life. The study and initial analysis of ideas, implementation of production processes and their placement on the market, give the Business Economics subject, highly complementary and very important for student technical training, received by this degree. The critical observation of the situation of Business Economics teaching, has led to propose a project of educational innovation that meets the needs of learning in entrepreneurship, solving the problems of teaching, while the transversal competences CT-4 are incorporated "innovation, creativity and entrepreneurship"and CT-6" Teamwork and leadership ". Considered fundamental in the performance of management. The competencies CT-03 "Analysis and Troubleshooting," and CT-12 "Planning and time management" fostering a better learning process.
\end{abstract}

Keywords: competencies, business idea, entrepreneurship, case study, simulation

\section{Resumen}

El proceso de construcción del Espacio Europeo de Educación Superior (EEES) ha supuesto una nueva organización de las enseñanzas universitarias en sus diferentes niveles, con un cambio en la orientación de la enseñanza, pasando de centrarnos en el aprendizaje de contenidos, a una orientación completamente diferente basada en resultados de aprendizaje y desarrollo de competencias. La carrera de diseño forma a profesionales en la vertiente científica y técnica para el desarrollo de nuevos diseños y productos desde la generación de las ideas, la producción, la fabricación y el posterior lanzamiento, incluido el estudio del impacto ambiental al final de su vida útil. La asignatura de Economía de la Empresa adquiere una condición muy 
relevante como complemento de la formación técnica que reciben los alumnos de este grado. La observación crítica de la situación de la docencia de empresa, ha llevado a platear un proyecto de innovación docente que dé respuesta a las necesidades de aprendizaje en emprendimiento, a la vez que atiende los problemas de docencia, y se incorporan las competencias transversales CT-4 "Innovación, creatividad y emprendimiento", y CT-6 "Trabajo en equipo y liderazgo". Consideradas como fundamentales en el desempeño de la gestión empresarial. También las competencias CT-03 "Análisis y resolución de problemas", y CT-12 "Planificación y gestión del tiempo" que favorezcan un mejor proceso de aprendizaje.

Palabras clave: competencias, idea de negocio, emprendimiento, estudio de caso, simulación

\section{Introducción}

La carrera de diseño tiene por objeto formar científica y técnicamente a profesionales para que sean capaces de dirigir y gestionar todo el proceso de vida de un producto desde la generación de ideas (análisis de mercados, marketing, diseño básico, ...) pasando por la producción, la fabricación y el lanzamiento del producto, hasta el estudio del impacto ambiental al final de su vida útil. El estudio y análisis inicial de las ideas, la implementación de los procesos de producción de nuevos productos y proyectos técnicos, y su colocación en distintos segmentos o nichos de mercado, otorgan a la asignatura de Economía de la Empresa una condición muy relevante, altamente complementaria a la formación técnica que reciben los estudiante de este grado.

Los objetivos de la asignatura de "Empresa” son:

a) que el alumno comprenda la importancia de la empresa como unidad económica de producción, generadora de nuevos diseños de productos, que implementa los procesos necesarios para la fabricación y producción de los mismos y también responsable de su comercialización,

b) que el alumno comprenda los mecanismos de funcionamiento económico de la empresa, considerando sus principales unidades funcionales o subsistemas: sus funciones, y sus interrelaciones,

c) que el alumno conozca y sea capaz de utilizar técnicas de gestión empresarial en la toma de decisiones.

Hay que pensar que desde la generación de las idea hasta que se implementan los procesos de producción correspondientes para la fabricación y la puesta en el mercado, es necesario un análisis en profundidad de la idea original de diseño, desde la perspectiva de la viabilidad económica, que nos permita determinar si esa idea es una verdadera idea de negocio. Y ello incluye, desde la generación de los diseños para cubrir un nicho, vacío o necesidad no satisfecha, y la existencia de tecnología que los haga posible técnicamente, hasta el análisis de la viabilidad económica. En el contexto actual de mercados globalizados, los aspectos económicos adquieren una gran relevancia, pues no sólo debemos preocuparnos de que los 
diseños cumplan la función para la que se propusieron (eficacia), sino que además debe realizarse al mejor coste y con el mejor uso de los recursos (eficiencia).

Sin embargo la relevancia de los aspectos económicos en el diseño de nuevos productos, contrasta con el escaso peso en carga docente que tiene la formación en empresa, y el hecho que se desarrolle en cursos iniciales. Por otra parte, los recursos que se destinan a la docencia (profesorado, aulas, dispositivos digitales, ...) definen una realidad para el desarrollo de la docencia, que debe tomarse en consideración. Los grupos son relativamente numerosos, normalmente en torno a los 40 alumnos y en las aulas no se dispone de un ordenador para cada alumno, compartiéndolos por parejas.

Las investigaciones en torno a la propia práctica docente y sus consecuencias en el aprendizaje de los alumnos, son las que llevan habitualmente a las propuestas de innovación docente. La identificación de un problema específico de aprendizaje o de un dilema docente (¿hago esto o lo otro?) deben activar procesos de investigación en el aula para examinar y mejorar el aprendizaje de los alumnos (Morales, 2012). El fin último será adaptar la docencia a las circunstancias de aprendizaje, buscando el mejor resultado en el proceso de aprendizaje.

La Universitat Politècnica de València (UPV) ha impulsado en los últimos años, a través de la iniciativa "Docencia en Red", la integración de la plataforma de aprendizaje PoliformaT. Un entorno virtual de aprendizaje, soporte para la enseñanza presencial pero también para la impartición de cursos on-line, que incluye un conjunto muy completo de herramientas, para el suministro de material al alumno, la interacción profesor-alumno, la programación, la evaluación y la gestión de las asignaturas (Martínez Rubio, y otros, 2010). Herramienta incuestionable y fundamental en la docencia de cualquier materia, y elemento básico en el desarrollo de cualquier proyecto educativo, como lo ha sido en el desarrollo del proyecto que se describe en el presente documento.

Por otra parte, el Espacio Europeo de Educación Superior (EEES) ha traído consigo un cambio en la orientación de la enseñanza, marcando un nuevo paradigma de aprendizaje en la educación superior pasando de centrarnos en el aprendizaje de contenidos, a una orientación completamente diferente basada en resultados de aprendizaje y desarrollo de competencias (Sanabria-Codesal, y otros, 2014). En la actualidad la UPV se encuentra implantando un modelo de formación en competencias transversales consideradas necesarias para el desempeño profesional de los titulados de la UPV, al margen de las competencias técnicas. Modelo que debe incorporarse en el diseño de las propuestas educativas.

Actualmente, también la UPV, ha impulsado la aplicación de la metodología docente del flip teaching o aprendizaje inverso. La metodología se basa en la preparación anticipada por parte del alumno de las materias, a partir de materiales que facilita el profesor, de forma programada, para utilizar la clase presencial con posterioridad como sesión para la resolución de dudas y afianzamiento de conocimientos, y la realización de ejercicios prácticos. 
Bajo la visión metodológica de action research (investigación-acción) ${ }^{1}$, y ante la observación crítica de la situación de la docencia de empresa (carga docente escasa, en cursos iniciales, grupos numerosos, limitaciones en el acceso a recursos informáticos para tod@s obligando a compartir los recursos por parejas), se plantea un proyecto de innovación docente que atienda a las necesidades de aprendizaje en emprendimiento, resolviendo los problemas de docencia, a la vez que se incorporan las competencias transversales CT-4 "Innovación, creatividad y emprendimiento”, y CT-6 “Trabajo en equipo y liderazgo”. Consideradas como fundamentales en el desempeño de la gestión empresarial. También las competencias CT-03 “Análisis y resolución de problemas”, y CT-12 "Planificación y gestión del tiempo" que favorezcan un mejor proceso de aprendizaje. Además la asignatura está inmersa en un proyecto docente, al margen de la propuesta de innovación que aquí se presenta, de incorporación de la metodología del flip teaching con el objeto de garantizar el alcance de contenidos propuestos para las sesiones de prácticas de la asignatura. Los resultados resultados de este proyecto docente no se contemplan en el presente estudio.

\section{Objetivo}

El objetivo de la presente comunicación es presentar una propuesta de docencia que contribuya a la mejora del aprendizaje de los aspectos de viabilidad de los nuevos diseños y proyectos de ingeniería, interconectando los contenidos de aprendizaje de carácter técnico con los relativos al estudio de la viabilidad económica, dotando a los alumnos de las herramientas necesarias para el estudio y análisis de las propuestas de nuevos diseños, e incorporando a la perspectiva de la idea de diseño, la idea de negocio.

\section{Desarrollo de la innovación}

La baja carga docente ha condicionado la organización de los contenidos de la asignatura de Empresa, en el grado de Diseño. Con el objeto de dotar a los alumnos de los conocimientos y las técnicas de análisis de viabilidad de los diseños de nuevos proyectos, las sesiones de prácticas se centran en las técnicas de evaluación financiera de proyectos de inversión, para que los alumnos conozcan herramientas en la toma de decisiones de inversión, y en las técnicas de análisis de las fuentes de financiación para que los alumnos conozcan herramientas en la toma de decisiones de financiación de los proyectos. Aportando en las sesiones de prácticas los contenidos teóricos y los desarrollos prácticos para la adquisición de estos conocimientos.

\section{Trabajo en equipo}

Los grupos de prácticas son muy numerosos, y el tamaño de las aulas de prácticas obliga a los alumnos a compartir los recursos informáticos. Aprovechando esta situación, se ha optado

\footnotetext{
${ }^{1}$ Una orientación metodológica de investigación para mejorar los procesos de enseñanza-aprendizaje. Se trata de un enfoque en el que a partir de una reflexión crítica sobre la situación actual se suceden de manera cíclica fases de puesta en práctica de algún tipo de innovación y de reflexión sobre los resultados que pueda llevar a una solución práctica, en la docencia de esta asignatura.
}

(c) EY-NC-ND 2016, Universitat Politècnica de València 
por el trabajo en equipo para introducir de manera efectiva la competencia trasversal CT-6 “Trabajo en equipo y liderazgo".

La competencia incluye la dimensión del trabajo colaborativo o en equipo, y el desarrollo de la capacidad de liderazgo. Trabajar y liderar equipos de forma efectiva para la consecución de objetivos comunes, y contribuir al desarrollo personal y profesional de estos. Ambas están interrelacionadas, dado que el éxito del trabajo en equipo precisa de liderazgo y, por otra parte, no puede existir liderazgo si no hay equipo. Algunos autores diferencian en el trabajo en equipo distintos modelos. El modelo de trabajo cooperativo que pretende superar los conflictos y dificultades de aprendizaje debidos a las diferencias, con asistencia mutua, en las que uno de los miembros ejerce el liderazgo contribuyendo al aprendizaje de los otros, mientras que el modelo colaborativo, ayuda al estudiante a enfrentar su autonomía, no existe un líder, sino que cada uno actúa como líder en la tarea que le ha designado el grupo. Todos los integrantes del grupo son líderes y evaluadores de los conceptos que se exponen (Pastor, 2007) (Blasco Magraner, y otros, 2013).

Por las circunstancias del propio proceso de aprendizaje, en el contexto de enseñanzas de grado universitarias, es el modelo colaborativo el que primará: grupo con reparto de tareas equitativo, comunicación entre los miembros fluída, recomendando a los alumnos la búsqueda de sinergias y complementariedad para la conformación de grupos heterogéneos, que comprendan e interpreten los contenidos de aprendizaje de forma compartida, pero que a la vez fomente el liderazgo individual, de manera que cada miembro del grupo sea su propio líder y responda por las cuestiones que le son encomendadas por el grupo. Resultando en beneficios valiosos para el grupo, y para cada miembro, que reforzará sus conocimientos y sus argumentaciones.

Existen tres formas de poner en práctica el aprendizaje colaborativo según Tudge (1994) y una de ellas es la interacción de pares. Por otra parte, la conformación de los equipos de trabajo es una tarea básica. Cuando se consigue que un equipo de trabajo -lo que denominamos equipo de base- funcione, no conviene modificar su composición. Por lo tanto, una de sus principales características, es que los equipos de base sean estables y perduren a lo largo de un curso e incluso de todo el ciclo formativo (Maset, 2003). Por ello, a diferencia de años anteriores, que las parejas no eran fijas y se podían modificar en cada sesión, se ha optado por que las prácticas se realicen por parejas, formadas al inicio del curso, que se mantienen inamovibles durante todas las sesiones.

Para instruir y ayudar a los alumnos al buen desarrollo de las sesiones de aprendizaje, se crea una "Guía de Buenas Prácticas para el Trabajo en Equipo" que se presentará en un Taller Inicial - "Taller 0". Los alumnos la tendrán a su disposición durante el curso, entre los recursos de prácticas, en PoliformaT. Incluye recomendaciones para la formación de los grupos, la importancia del compromiso y la responsabilidad para con los compañeros del equipo, para la preparación del trabajo, y para el propio desarrollo del trabajo en el aula. En el Taller inicial, y tras la presentación de la asignatura, la descripción del funcionamiento de

\footnotetext{
2 Llevada esta definición al terreno educativo, podemos entender como "buenas prácticas”, las acciones desarrolladas en el ámbito educativo que facilitan el aprendizaje (Gutiérrez Esteban, y otros, 2011)
}

(cc) EY-Nc-ND 2016, Universitat Politècnica de València

Congreso In-Red (2016) 
las prácticas a lo largo del curso a través de la formación de los equipos, y la presentación de la Guía de Buenas Prácticas, cada alumno se someterá a un test de autoevaluación para que reflexione y evalúe su actitud y aptitud para el liderazgo y el trabajo en equipo (Rúbrica en Anexo I). Al finalizar las sesiones de prácticas, al final del curso, cada alumno se someterá nuevamente al mismo test de autoevaluación, para que cada alumno reflexione sobre si ha cambiado su actitud frente al compañero. Y cada alumno, se someterá a evaluación por su compañero (pareja de equipo). Comparándose los resultados de ambas con la evaluación inicial. En ningún caso los resultados de las evaluaciones del trabajo en equipo se utilizarán para calificar las prácticas, solo se tendrá en cuenta la evaluación del trabajo académico realizado a lo largo del curso en las distintas sesiones.

\section{Tabla 1 Objetivos y Resultados de aprendizaje planteados para implementar la CT-6 "Trabajo en equipo y liderazgo"}

\section{Objetivos:}

- Formar al alumnado sobre los conceptos de trabajo en equipo y liderazgo para fomentar en ellos estas capacidades.

- Concienciar sobre los tipos de liderazgo asumidos en el trabajo en equipo, y su influencia en las relaciones interpersonales y los resultados del trabajo en grupo.

- Constatar y evaluar los roles asumido por el alumnado a través de un test de liderazgo y un cuestionario de autoevaluación.

\section{Resultados de aprendizaje:}

- Participar y colaborar activamente en las tareas de equipo y fomentar la confianza, la cordialidad y la orientación a la tarea conjunta.

- Contribuir a la consolidación y desarrollo del equipo favoreciendo la comunicación, el reparto equilibrado de tareas, el clima interno y la cohesión.

- $\quad$ Dirigir grupos de trabajo, asegurando la integración de los miembros.

Fuente: Documento UPV “Competencias Transversales”

\section{Emprendimiento}

Como ya se indicaba en la introducción de la presente comunicación, el propio objetivo de formación del Grado de Diseño, requiere contenidos de aprendizaje para el desarrollo de las ideas, que debe incluir de forma incontestable el estudio de la idea desde el punto de vista de la viabilidad económica y la idea de Negocio, proporcionando información relevante, recursos y herramientas para el emprendimiento.

Con el fin de incorporar en el desarrollo de los proyectos de nuevos diseños, junto a los aspectos relativos a la viabilidad técnica, otros aspectos relativos a las necesidades y atributos de los nuevos productos considerando variables relativas a la viabilidad económica y comercial, se ha propuesto el desarrollo de un “Taller 1_ Idea\&Idea de Negocio”. El Taller introduce al conocimiento de herramientas de evaluación preliminar de una idea o proyecto de diseño, para evaluar diferentes factores relacionados con el potencial de su idea/proyecto, conocer el grado de madurez de la idea, y también los resultados de económicos y/o de 
rentabilidad de la misma, que la convierten o no en una idea de negocio. Los contenidos de la práctica están recogidos en un guión de la prácticas. Los alumnos lo tendrán a su disposición durante el curso, entre los recursos de prácticas, en PoliformaT. El Taller, a través de la presentación de ejemplos concretos de buenas ideas de diseño que son además buenas ideas de negocio, propone el análisis de estas ideas (Anexo II: Ficha de Análisis), así como insta a los alumnos a la elección de propuestas de diseño como iniciativas de emprendimiento simulado, que se utilizarán en los Talleres de las sesiones posteriores de prácticas. Se pretende así introducir al alumno en las dinámicas de emprendimiento, dotándolo de herramientas para el análisis de las ideas de diseño, que permitan la toma de decisiones sobre la viabilidad comercial de las mismas y su proyección empresarial.

El objetivo del proyecto de educativo al introducir este "Taller_1" es estimular la iniciativa y la proactividad en el aula para fomentar el emprendimiento empresarial.

Tabla 2 Objetivos y Resultados de aprendizaje planteados para implementar la CT4-
"Innovación, Creatividad y Emprendimiento"

\section{Objetivos:}

Fomentar en el alumno el pensamiento y análisis y la posibilidad de abordar la realidad desde otras perspectivas que favorezca nuevos diseños que resulten en innovación

Formar al alumno para que sea capaz de analizar los resultados de la innovación

Favorecer una mejor comprensión e integración de los contenidos docentes del grado relativos a empresa, de cara al desarrollo de actitudes y aptitudes emprendedoras, creatividad, orientación a la acción, y consideración de la capacidad de riesgo

Favorecer una formación más completa e integral del profesional ingeniero, con una visión más global de la formación, orientada al desempeño profesional, que le permitan una mejor y más inmediata aplicación de los conocimientos adquiridos

Fomentar la voluntad de asumir responsabilidades, la proactividad e iniciativa y la toma de decisiones, a partir de una mejor formación en herramientas de planificación y previsión

Llevar al campo del diseño, de una manera aplicada, conocimientos de gestión y organización de empresa, de modo que los alumnos percibieran su sentido y su aplicación futura en la experiencia profesional

Dotar a los alumnos de mejores herramientas de análisis en el desarrollo de proyectos de nuevos diseños, abarcando los elementos de viabilidad de una forma global e integral, y con el análisis de resultados económicos

Formar al alumno en los conceptos de emprendimiento, y los aspectos relativos a la idea y el análisis de la misma desde el punto de vista de la idea de negocio

Acercar a los alumnos al mundo empresarial, y la toma de decisiones

\section{Resultados de aprendizaje:}

Surgimiento de nuevas ideas o alternativas innovadoras a situaciones o problemas que se plantean al alumno

Generar ideas originales y de calidad

(cc) EY-NC-ND 2016, Universitat Politècnica de València

Congreso In-Red (2016) 
- Familiarizar al alumno en los conceptos de idea de diseño y las posibilidades de viabilidad que la convierten en idea de negocio

- Sentar las bases para desarrollar análisis económicos de las ideas de diseño y la determinación de la oportunidad como ideas de negocio, a partir de los resultados analizados, y la toma de decisiones coherentes con los resultados del análisis.

Fuente: Documento UPV “Competencias Transversales”

\section{Análisis y resolución de problemas, y Planificación y gestión del tiempo}

Ambas competencias, CT-03 “Análisis y resolución de problemas”, y CT-12 “Planificación y gestión del tiempo”, se implementan a través del desarrollo de los restantes “Talleres 2 a 6”. Los cinco talleres se han propuesto a partir de un posible Idea de Negocio, definida a partir de los resultados del Taller 1 de emprendimiento, como resultado del análisis de las propuestas de innovación realizadas por los alumnos. Los “Talleres_2 a 5”, se estructuran en cinco sesiones en las que el alumno aprenderá técnicas de evaluación de los proyectos de innovación, analizando la viabilidad de los mismos desde la perspectiva de la inversión y las herramientas de análisis que permiten determinar la viabilidad de la misma (índices de rentabilidad), y posteriormente las técnicas de análisis de las fuentes de financiación para que los alumnos conozcan herramientas en la toma de decisiones sobre fuentes de financiación de los proyectos, permitiendo determinar el coste de las fuentes alternativas. Aportando en las sesiones de prácticas los contenidos teóricos y los desarrollos prácticos para la adquisición de estos conocimientos.

Con el objeto de incorporar la competencia CT-03 “Análisis y resolución de problemas”, y CT-12 "Planificación y gestión del tiempo":

- los desarrollos prácticos en estas sesiones se basarán en problemas prácticos relativos al caso concreto definido como iniciativa de emprendimiento simulado, a partir del “Taller_1”.

- $\quad$ los enunciados de los problemas deben facilitar la formación y el desarrollo de conceptos, y permitir identificar de forma clara el resultado que se busca

- $\quad$ se debe atender especialmente el procedimiento de resolución, introduciendo en el conocimiento de herramientas para dicha resolución, así como la interpretación y el análisis de los resultados de las mismas.

- $\quad$ deben permitir extraer conclusiones importantes para la toma de decisiones. En este sentido las aportaciones diversas que supone el trabajo en equipo resultan muy enriquecedoras 

resolución de Problemas” y la CT4- “Planificación y Gestión del Tiempo"

\section{Objetivos:}

Desarrollar en los alumnos una actitud mental mediante la aplicación de procedimientos estructurados de resolución de problemas que promueva su capacidad de aprender, comprender y aplicar conocimientos de forma autónoma

Contribuir al desarrollo de otras competencias básicas como el trabajo en equipo, la creatividad, el análisis o el liderazgo

Favorecer que el alumne incorpore en la resolución de los problemas las tareas previas de identificación, y de programación y planificación de las actuaciones a desarrollar para la resolución de los mismos

\section{Resultados de aprendizaje:}

Identificar y analizar un problema para generar alternativas de solución, aplicando los métodos aprendidos: definir con exactitud el problema a resolver, recopilar la información significativa para la solución

Utilizar la experiencia y el criterio para analizar las causas de un problema y construir una solución más eficiente y eficaz: identificar bien las causas que generan el problema, identificar las posibles alternativas para la solución, evaluar ventajas y desventajas de cada alternativa, decidir la solución oportuna, decidir la aplicación y el seguimiento de la misma

Definir claramente los objetivos a cumplir, establecer un orden de prioridades para la resolución de los mismos, y programar y planificar las actividades para la resolución

Fuente: Documento UPV “Competencias Transversales”

Para trabajar las competencias en cuestión, se desarrollaron los “Talleres_2 a 6”, mediante el planteamiento de cuestiones relativas al estudio de un caso concreto de iniciativa de emprendimiento, ligado con la práctica anterior, "Taller_1”, analizando la viabilidad económica de la idea como proyecto de inversión, y después, una vez decidida la oportunidad y viabilidad de la idea de negocio, aprender cuestiones relativas a las alternativas de financiación, y como estudiarlas. Planteando las prácticas en conjunto, simulando el desarrollo de las tareas de gestión de la empresa, considerando la interconexión real existente entre las distintas decisiones a tomar relativas a inversión-financiación. La utilización de las simulaciones presenta el beneficio en el aprendizaje de los alumnos, de la facilidad de comprensión de los conceptos teóricos y de los procesos a estudiar. A la vez que el aprendizaje mediante el estudio de casos permite llevar al contexto o complejidad global de la toma de decisiones, un caso particular y concreto (Álvarez, y otros, 2012), ayudando al alumno en el entendimiento del contexto global, y la interpretación de los resultados y las conclusiones que se pueden extraer en la resolución de cada problema.

Los alumnos cuentan con guiones de prácticas para cada una de las sesiones o talleres disponibles desde PoliformaT. En estos guiones se plantean los problemas de decisión de la empresa que durante la sesión de prácticas se resolverán. En las sesiones de prácticas los grupos realizarán un trabajo tutelado por el profesor, desarrollando cada grupo sus propias herramientas informáticas para la resolución del ejercicio, en Excel. En las sucesivas sesiones el grupo irá creando su propio libro de Excell, que se conformará a modo de plantilla de 
análisis, válida para el análisis de cualquier idea-proyecto, con el objeto de que el alumno se cree una herramienta de análisis útil para su desarrollo profesional futuro.

La interpretación y el análisis de los resultados, para extraer conclusiones la pondrá en práctica cada alumno de forma individual al contestar un test al final de la sesión. El Test es de autoevaluación, generado en poliformaT, a través de una batería de preguntas que aleatoriza las preguntas y el orden de las respuestas, generando para cada alumno un test completamente distinto al de sus compañeros.

El desarrollo de cada sesión de prácticas, con el archivo de la Hoja de Cálculo de cada grupo en el espacio compartido, y la realización de los Test al final de la sesión, permiten comprobar a través de dos rúbricas (Anexo III y Anexo IV) las competencias CT-3 "Análisis y Resolución de Problemas” y la CT-12 "Planificación y Gestión del Tiempo". La evaluación se realiza al final del curso, para cada grupo.

\section{Conclusiones}

El Espacio Europeo de Educación Superior (EEES) ha comportado un cambio en la orientación de la enseñanza, antes centradas en el aprendizaje de contenidos, para orientarse hacia los resultados de aprendizaje y desarrollo de competencias.

La carrera de diseño tiene por objeto formar científica y técnicamente a profesionales para que sean capaces de dirigir y gestionar todo el proceso de vida de un producto desde la generación de ideas (análisis de mercados, marketing, diseño básico, ...) pasando por la producción, la fabricación y el lanzamiento del producto, hasta el estudio del impacto ambiental al final de su vida útil. El estudio y análisis inicial de las ideas, la implementación de los procesos de producción de nuevos productos y proyectos técnicos, y su colocación en distintos segmentos o nichos de mercado, otorgan a

La asignatura de Economía de la Empresa constituye una materia altamente relevante, complementaria a la formación científica y técnica de los estudiante del grado de diseño. Partiendo de la idea de diseño o de nuevo productos, las herramientas que aporta la asignatura de Empresa, permien al profesional, el estudio y análisis de las mismas, desde el punto de vista de la rentabilidad y la oportunidad de negocio de las mismas, orientándolo hacia la idea de negocio, y el análisis de las ideas desde el punto de vista de la viabilidad comercial.

La observación crítica de la situación de la docencia de empresa (carga docente escasa, en cursos iniciales, grupos numerosos, limitaciones en el acceso a recursos informáticos para tod@s obligando a compartir los recursos por parejas), ha llevado a platear un proyecto de innovación docente que contempla las necesidades de aprendizaje en emprendimiento, resuelve en parte los problemas de docencia, a la vez que incorpora las competencias transversales CT-4 "Innovación, creatividad y emprendimiento”, y CT-6 “Trabajo en equipo y liderazgo". Consideradas como fundamentales en el desempeño de la gestión empresarial. También las competencias CT-03 “Análisis y resolución de problemas", y CT-12 "Planificación y gestión del tiempo" para favorecer un mejor proceso de aprendizaje. 


\section{Referencias}

Álvarez Carmen y Maroto José Luis San Fabián La elección del estudio de caso en investigación educativa [Publicación periódica] // Gazeta de Antropoligía. - 2012. - 14 : Vol. 28/1. - pág. http://hdl.handle.net/10481/20644.

Blasco Magraner Salvador y Bernabé Valero Gloria ¿ Cómo desarrollar la competencia colaborativa en el alumnado universitario? Una Propuesta de implementación y evaluación. [En línea]. - 2013. - http://m.web.ua.es/va/ice/jornadas-redes/documentos/2013posters/335175.pdf.

Gutiérrez Esteban P [y otros] Buenas prácticas en el desarrollo de trabajo colaborativo en materias TIC aplicadas a la educación [Publicación periódica] // Profesorado. Revista de currículum y formación del profesorado. - 2011. - 1 : Vol. 15.

Martínez Rubio Juan Miguel, Ramírez Blanco Manuel Jesús y Ferrando Bataller Miguel Universidad Nacional Autónoma de México [En línea] = Docencia en Red, una apuesta estratégica en la Universidad Politécnica de Valencia. // Reposital. Material educativo.. - Octubre de 2010. - http://hdl.handle.net/123456789/1203.

Maset Pere Pujolàs El aprendizaje cooperativo: algunas ideas prácticas. [Informe]/ Universidad de Vic.. - 2003. - Documento de Trabajo.

Morales Pedro Investigación en Innovación Educativa [Publicación periódica] // Revista Iberoamericana sobre Calidad, Eficacia y Cambio en Educación. - 2012. - 2 : Vol. 8. http://www.rinace.net/reice/numeros/arts/vol8num2/art3.pdf.

Pastor María Luisa Carrió Ventajas del uso de la tecnología en el aprendizaje colaborativo. [Publicación periódica] // Revista Iberoamericana de Educación. - 2007. - 4 : Vol. 41. - pág. 5 .

Sanabria-Codesal Esther [y otros] Análisis de las Dimensiones Competenciales Incluidas en Diferentes Asignaturas en Ingenierías [Conferencia] // Jornadas de Innovación Educativa y de Docencia en Red / ed. Valencia Universidad Politécnica de. - Valencia : Universidad Politécnica de Valencia, 2014. - págs. 1050-1064.

Tudge A Vigotsky: la zona de desarrollo próximo y su colaboración en la práctica de aula. [Publicación periódica] // Universidad de Cambridge. - [s.l.] : Universidad de Cambridge, Nueva York, 1994. 